\title{
DE-F9S08-94NV/1630
}

\section{GAMMA RAY DETECTOR USING GALLIUM ARSENIDE TO DEVELOP AN ELECTRODE DETECTOR}

\author{
Final Technical Report
}

For the Period September 30, 1994 to November 16,1997

Glenn F. Knoll

Nuclear Engineering \& Radiological Sciences

College of Engineering

University of Michigan

Ann Arbor, Michigan 48109

March 26, 1999

Prepared for

THE U. S. DEPARTMENT OF ENERGY

AWARD No. DE-FG08-94NV11630 


\section{DISCLAIMER}

Portions of this document may be illegible in electronic image products. Images are produced from the best available original document. 
The emphasis of the DOE-funded detector project at the University of Michigan has been on the improvement of the performance of room-temperature gamma ray spectrometers. We have concentrated on the material known as CZT, a blend of cadmium and zinc tellurides, as the material of primary interest. The bandgap and leakage current from this material are appropriate for use of large volumes at room temperature.

The major drawback of this material is poor hole transport. Our principle effort has been to overcome this problem by devising spectrometer configurations that do not depend on the collection of holes for good performance. The method of choice has been the use of coplanar electrodes of our unique design that carefully balance the weighting potentials of the two independent sets of anode electrodes to give a uniform response throughout the detector volume. We have demonstrated through a series of technical papers that by modulating the strip widths appropriately, the weighting potential for the difference signal can be made uniform enough to permit energy resolutions below $1 \%$.

Our best results to date have been the achievement of an energy resolution of $1.8 \%$ and $1.9 \%$ on two.CZT crystals, each of 1 cubic centimeter volume. These results still stand as the best energy resolution obtained anywhere in the world in CZT spectrometers of this volume. We feel that there is ample room for improvement, since these results were obtained with patterns that were one generation before the optimized design that we have now worked out.

In addition to providing excellent spectroscopic performance, the coplanar grids also permit a unique type of diagnostics to be performed on the nature of the electron transport within the crystals. In conventional detectors, the induced charge depends on the product of the number of charge carriers multiplied by the distance that the travel from their point of origin. The two factors cannot easily be separated. In a coplanar detector, two independent signals can be derived: one from the coplanar anode difference signal, and the other from the cathode or opposite surface. The cathode signal has the same characteristics as that from a conventional detector, and thus depends on the product of charge and distance. The coplanar signal, however, has the unique property that it depends only on the absolute charge. Thus by taking the ratio of the two signals, we derive a depth coordinate. We have demonstrated the ability to separate the measured pulses into those arising from 20 independent layers that are parallel to the anode surface and cover the entire volume from anode to cathode. This depth sensing allows us to make corrections for electron trapping that takes place in all crystals, regardless of their present quality.

The depth signal also allows us to measure some of the charge transport properties of the crystal in a unique way. Since we can track the depth of a single electron as a function of time, we are able to find its drift velocity and thus the mobility as an absolute measurement. Furthermore, by studying the decrease in the coplanar anode signal as a function of depth, we can measure the product of the mobility and lifetime of the electron. Again, by taking the ratio of the two signals, we obtain an independent measure of the charge lifetime to help characterize the material. These measurements can also be carried out as a function of depth to study the nonuniformities that are present in the crystal. This type of diagnostic ability will be of value in helping evaluate CZT crystals obtained from various sources in the future. 